\title{
Uropathogens and Diabetes Mellitus- a perspective
}

\author{
Dhandapany Senthil Pragash ${ }^{1}$, Smiline Girija $^{2}$, Usha Sekar $^{1}$, Vijaya Rayapu ${ }^{1}$ \\ D.S.Sheriff ${ }^{1}$ \\ Department Of Microbiology, Melmaruvathur Adhiparasakthi Institute Of Medical Sciences And Research, \\ (Affiliated To Tamilnadu Dr MGR Medical University, Chennai), Melmaruvathur, Kancheepuram District, \\ Tamilnadu - 603319, India. \\ Department Of Microbiology, Meenakshi Ammal Dental College, (Affiliated To MAHER University), \\ Maduravoyal, Chennai - 600095, India.
}

\section{Introduction}

Urinary tract infections are defined as diseases which are caused by a microbial invasion of the genitourinary tract, which extends from the renal cortex of the kidney to the urethral opening. They represent the most commonly acquired bacterial infections and they account for an estimated $25-40 \%$ of the nosocomial infections [1].

Urinary tract infections are "uncomplicated" when they occur in a normal urinary tract with no structural, functional or underlying host illness to account for the infection, or "complicated" when an underlying abnormality is thought to have enabled the infection to occur [2]. The incidence of UTI as a result of viral or fungal infection is considered to be rare phenomena. Though the infection seems to be harmless in the initial stages, the patient shows a variety of symptoms as the stage progresses and can lead to death in severe circumstances. Research studies have defined urinary tract infection as the most common form of bacterial infection [3]. The predominant pathogen responsible for UTI is E. coli which constitutes up to $80-85 \%$ and is followed by Staphylococcus saprophyticus which accounts to 5-10\%. In addition to the above mentioned bacterial species, Klebsiella, Proteus, Pseudomonas and Enterobacter are associated with UTI. The bacteria enter the bladder through urethra and the infection can also occur through blood and lymph [4].

The clinical manifestation of UTI depend upon the portion of the urinary tract involved, the etiologic organism, the severity of the infection and patients ability to mount an immune response to it. Signs and symptoms include fever, dysuria, and urinary urgency, cloudy or fragrant urine. UTI is an extremely common condition that occurs in both male and female [5]. UTI can affect lower and sometimes both lower and upper urinary tracts. The term cystitis has been used to define the lower UTI infection and is characterized by symptoms such as dysuria, frequency, urgency, and suprapubic tenderness. The presence of the lower UTI symptoms does not exclude the upper UTI which is often present in most UTI cases [6]. Bacteriuria, or the presence of bacteria in urine, is associated with both asymptomatic and symptomatic urinary tract infection and underpins much of the dynamic of microbial colonization of the urinary tract [7].

Urinary tract infection or UTI is said to exist when a significant number of microorganisms, usually greater than 105 cells per millilitre of urine, are detected in properly collected mid-stream "clean catch" urine [8]. There is evidence that patients with diabetes have an increased risk of asymptomatic bacteriuria and urinary tract infections (UTIs). UTI is the most common bacterial infection in diabetic patients [9].An association between urinary tract infection (UTI) and diabetes mellitus was noted in an autopsy series reported in the 1940s. The urinary tract is the principal site of infection in diabetes. Changes in host defense mechanisms, the presence of diabetic cystopathy and of microvascular disease in the kidneys may play a role in the higher incidence of UTI in diabetic patients [10].

Diabetes mellitus has a number of effects on genitourinary system. Urinary Tract Infection (UTI) is more common in diabetics because of a combination of host and local risk factors. Under some circumstances urine may be inhibitory or even bactericidal against uropathogens. Modification of chemical composition of urine in diabetes mellitus can alter the ability of urine and support the growth of microorganisms. Autonomic neuropathy in diabetes mellitus impairs bladder emptying and subsequent urological manipulation predispose to UTI [11].

The reason for this predisposition is not completely understood. Studies are limited, however, evidence of some reports have shown that diabetes affects many systems that protect against infection in general and against urinary tract infections specifically. Poor circulation of blood in diabetes reduces the ability of infection fighting white blood cells to get to their target site, even when they do get there, they are less able to ingest the offending bacteria and kill them than normal white blood cells. Many people with diabetes also have dysfunctional bladders that contract poorly. This allows urine to remain in static pools for long periods of time, providing ponds for bacteria to grow in [12]. 
Weakened leukocyte phagocytosis has been found in both Type 1 and Type 2 DM and explains the diminished infecting bacterial clearance during hyperglycemia as observed in mouse models. Phagocytosis was reduced by $50 \%$ in the leukocytes in diabetic mice infected by Staphylococcus aureus. The main factor of neutrophil dysfunction is the hyperglycemia, which alters neutrophil chemotaxis, phagocytic action and intracellular killing of the bacteria. Respiratory burst by monocytes from hyperglycemic patients has been affected. Humoral immunity in diabetic patients can be affected as well, which can be shown by a shorter duration of the protective antibodies after tetanus vaccination and lower levels of tetanus antitoxin compared with those in non-diabetic subjects. Alterations in levels of complement fractions can also be found. Hyperglycemia suppresses activation of $\mathrm{C} 3$ fraction, which results in changed $\mathrm{C} 3$ tertiary structure and impairs the interactions of C3 with the bacterial pathogens [13]. Decreased mobilization of polymorphonuclear leukocytes, chemotaxis, and phagocytic activity may occur during hyperglycemia.

The hyperglycemic environment also blocks the antimicrobial function by inhibiting glucose-6phosphate dehydrogenase (G6PD), increasing apoptosis of polymorphonuclear leukocytes, and reducing polymorphonuclear leukocyte transmigration through the endothelium. In tissues that do not need insulin for glucose transport, the hyperglycemic environment increases intracellular glucose levels, which are then metabolized, using NADPH as a cofactor. The decrease in the levels of NADPH prevents the regeneration of molecules that play a key role in antioxidant mechanisms of the cell, thereby increasing the susceptibility to oxidative stress [14]. Various studies done worldwide have shown changing patterns in the etiology of UTIs indiabetic patients [15].

In a cross sectional study conducted in Nepal to assess the spectrum of uropathogens and their antibiotic sensitivity pattern in diabetic patients, the overall culture positivity rate was $34.5 \%$. Escherichia coli was the most frequent organism $(64.5 \%)$ followed by Klebsiella sps $(22.6 \%)$. E. coli was highly sensitive to gentamicin and nitrofurantoin among the tested antimicrobials followed by cotrimoxazole, norfloxacin and ciprofloxacin. Least sensitivity rate was observed with ampicillin and cephalexin [16]. In another study conducted in Nepal to determine the prevalence of UTI among diabetic patients, the causative pathogens \& their antimicrobial susceptibility pattern the overall prevalence of UTI was found to be $54.76 \%$ from the total of 462 diabetic patients. The gram positive isolates and Escherichia coli were found to be sensitive to nitrofurantoin [17]. A prospective study done in India to know the bacteriological and resistance profile of isolates obtained from diabetic patients showed that 18 of $65(27.7 \%)$ had UTI. Twenty six bacterial species were isolated from urine samples. The most frequently isolated species was E.coli followed by K. Pneumoniae [18].

In a study conducted, for two years in Mysore (Southern India), to determine the prevalence and incidence of urinary tract infection among diabetic patients, about 900 samples were culture positive and 936 isolates were obtained out of 1085 urine samples. Escherichia coli was the major cause for urinary tract infection in both type $1(34 \%)$ and type $2(32 \%)$ diabetic patients, followed by Methicillin resistant Staphylococcus aureus (11.4\% and 12.6\%) [19]. In a study conducted in Karnataka, India, to determine the frequency of uropathogens and their

antibiotic susceptibility in different gender of diabetic patients found that from the total of 44 urine samples $68 \%$ of the patients were having asymptomatic bacteriuria. Biochemical characterization revealed prevalent gram negative organisms and E. coli as the predominant solate. Among the antibiotics tested, trimethoprim was found to be effective for empirical treatment of UTI and has covered the majority of urinary pathogens followed by nalidixic acid, Chloramphenicol and kanamycin. Most of the isolates were resistant to oxytetracycline [20]. In a prospective study carried out in Government Medical College Amritsar-India to find out theprevalence of uropathogens in diabetic patients and to study their antibiotic susceptibility pattern bacterial growth was obtained in $118(43 \%)$ cases out of the 270 urine specimens from diabeticpatients. Among these 50.84\% $(60 / 118)$ had asymptomatic bacteruria while symptomatic infection occurred in $49.15 \%(58 / 118)$ patients. Escherichia coli was the most frequent isolated (41.5\%), followed by Klebsiella species (14.4\%), Proteus species (10.1\%), Enterococci (8.4\%), Pseudomonas aeruginosa (6.7\%), Staphylococcus species (10.1\%) and Candida albicans $(8.4 \%)$. The isolates showed highest resistance against amoxicillin and ciprofloxacin, moderate resistance against norfloxacin, nitrofurantoin, gentamicin and cefotaxime while resistance against amikacin was low [21].

In a study done to assess prevalence of urinary tract infection and risk factors among Saudi patients with diabetes, the prevalence of UTI was $25.3 \%$ from the total of 1,000 diabetic patients and 7.2 and $41.1 \%$ in males and females, respectively [22]. In another study conducted in National Center of Diabetes- Baghdad, Iraq on 122 patients with diabetes mellitus to determine the prevalence of uropathogens and their antibiotic susceptibility significant bacteriuria was seen in 60(49.1\%) patients. Among the isolates 45 were gram negative bacilli and 15 were gram positive cocci. Among the gram negative bacilli E. coli was the predominant isolate (60\%) while Pseudomonas aeruginosa was the least (1.66\%). E.coli and Klebsiella pneumoniae showed highest sensitivity to piperacyclin followed by nitrofurantoin. Staphylococcus aureus had highest sensitivity to ciprofloxacin and cefoxitin (75\%) and the highest resistance to chloramphenicol (91.7\%). Most bacterial isolates 
exhibited complete resistance to Tetracyclin (100\%), only Enterobacter species had sensitivity to Tetracyclin (33.3\%) [21].

A study conducted at Buea and Limbe Regional Hospital Diabetic and Hypertensive Clinic in Cameroon, to investigate the prevalence of asymptomatic bacteriuria and UTIs in clinically diagnosed patients with diabetes and to determine the uropathogens responsible for ASB (asymptomatic bacteriuria) and UTIs as well as their antimicrobial susceptibility pattern, revealed that one hundred and two (81.6\%) of the total 125 urine samples had significant bacteriuria. Eight different bacteria were isolated from the study participants: Escherichia coli (48.0\%) were the most prevalent, followed by Staphylococcus aureus (19.6\%) and Proteus mirabilis $(8.9 \%)$. Most of the bacterial isolates were highly sensitive to gentamicin $(88.6 \%)$, imipenem $(87.9 \%)$, nitrofurantoin $(79.5 \%)$ and amikacin $(88.3 \%)$. Some of the bacterial isolates showed $53.3 \%$ resistance rate while all the bacterial isolates were highly resistant $(96.3 \%)$ to amoxicillin [22].

A cross sectional study conducted at Muhimbili National Hospital- Dar es Salaam, Tanzania to determine the prevalence and risk factors of bacteriuria in diabetic women and antimicrobial resistance pattern of the isolates revealed $13.7 \%$ (41/300) significant bacteriuria, of which $13.4 \%(31 / 231)$ and $14.5 \%(10 / 69)$ were asymptomatic and symptomatic bacteriuria, respectively. The isolated pathogens were Escherichia coli (39.0\%), Klebsiella pneumoniae (22.0\%), coagulase negative Staphylococci (14.65\%) and Proteus spp $(12.2 \%)$. Both Gram positive and negative bacteria showed high rate of resistance towards co-trimoxazole $(55.6 \%$ and $50.0 \%$, respectively). Gram negative bacteria showed high rate of resistance to ampicillin $(62.55 \%)$, penicillin (53.1\%) and moderate resistance to cefotaxime (18.8\%). Most uropathogens were resistant to cotrimoxazole, ampicillin and ciprofloxacin [23].

A prospective cross sectional study conducted in Gondar University Hospital, Ethiopia, on a total of 422 diabetic patients with asymptomatic UTI $(n=387)$ and symptomatic UTI $(n=35)$, to investigate for urinary tract infection showed $17.8 \%$ significant bacteriuria, of which $14.7 \%$ were asymptomatic and $51.4 \%$ were symptomatic. Out of the total 82 bacterial isolates, E. coli (31.7\%), coagulase negative staphylococci (CONs) (22\%), Klebsiella spp. (14.6\%),Enterococcus spp. (11\%) and S. aureus $(8.5 \%)$ were the commonest bacterial uropathogens inboth groups. All gram negative isolates showed intermediate level of resistance $(60-80 \%)$ against ampicillin and chloramphenicol. Low level of resistance $(<60 \%)$ was observed against amoxicillinclavulanic acid, ciprofloxacin, ceftriaxone, gentamicin and trimethoprimsulphamethoxazole. High level of resistance (>80\%) was observed against tetracycline. Gram positive bacteria showed low level of resistance $(<60 \%)$ to all antimicrobials tested except for tetracycline [24]. Therefore, it will be worthwhile to study and understand the common UTI present in diabetic patients of different regions and geographical locations. Such a study will help isolate the organisms which are locally unique to the diabetic patients for infection control and to undertake preventive measures. Multidrug-resistant Acinetobacter baumannii is a rapidly emerging pathogen in the health care setting, where it causes infections that include bacteremia, pneumonia, meningitis, urinary tract infection, and wound infection. The genus Acinetobacter is now defined as including gram-negative coccobacilli, with a DNA G+C content of 39 to $47 \mathrm{~mol} \%$, that are strictly aerobic, nonmotile, catalase positive and oxidase negative. Diabetic patients are 10 times more likely to develop Acinetobacter baumannii infections than the rest of the population. Carbapenems are considered one of the very few antibiotics left to treat infections caused by this organism.(25) The aim of this work was to characterise A. baumannii strains isolated from diabetic patients and to investigate whether there is a relationship between certain strains and low-levelcarbapenem resistance.

\section{References}

[1]. S. Niveditha, S. Pramodhini, S. Umadevi, Shailesh K, Selvaraj S. Isolation and the Biofilm Formation of Uropathogens in the Patients with Catheter Associated Urinary Tract Infections (UTIs). Journal of Clinical and Diagnostic Research. 2012; 6(9): 1478-

[2]. 1482

[3]. Mwaka AD, Mayanja-KH, Kigonya E, Kaddu-Mulindwa D. Bacteriuria among adult non-pregnant women attending Mulago ospital assessment centre in Uganda. African Health Sciences. 2011; 11(2): 182 - 9.

[4]. Shu'aibu Isa., Mohammed BM., Fatima Umar M., Abubakar Aisami et al. Prevalence andAntimicrobial Susceptibility Pattern of Uropathogenic Escherichia coli among Adult

[5]. Diabetic Patients Attending Federal Medical Centre Gombe, Gombe State Nigeria.Greener Journal of Microbiology and Antimicrobials. 2013; 1(1): 11-15.

[6]. Vasudevan R. Urinary Tract Infection: An Overview of the Infection and the AssociatedRisk Factors. J Microbiol Exp 2014; 1(2): $1-15$.

[7]. Arul PK C, KG Dileesh K, M Vijayan. A Cross Sectional Study on Distribution ofUrinary Tract Infection and their Antibiotic Utilisation Pattern in Kerala. International

[8]. Journal of PharmTech Research. 2012; 4(3): 1309-1316.

[9]. Wasnik DD, Tumane PM. Prevalence and antibacterial susceptibility pattern of UrinaryTract Infection Causing Human Pathogenic Bacteria. Asian Journal of Biomedical and

[10]. Pharmaceutical Sciences. 2012; 2(15): 1-3.

[11]. Ipe DS, Sundac L, Benjamin Jr WH, Moore KH, Ulett GC. Asymptomatic bacteriuria:prevalence rates of causal microorganisms, etiology of infection in different patient 
[12]. populations, and recent advances in molecular detection. FEMS Microbiol Lett 346.2013: 1-10.

[13]. Iregbu KC, Nwajiobi-Princewill PI. Urinary Tract Infections in a Tertiary Hospital in Abuja, Nigeria. Afr. J. Cln. Exper. Microbiol. 2013; 14(3): 169-173.

[14]. http://dx.doi.org/10.4314/ajcem.v14i3.9 (accessed 09 April 2015).

[15]. Chita T, Licker M, Sima A, Vlad Adrian, et al. Prevalence of Urinary Tract Infections in Diabetic Patients. Rom J Diabetes Nutr Metab Dis. 2013; 20(2): 099-105.

[16]. Janifer J, Geethalakshmi S, Satyavani K, Viswanathan V. Prevalence of lower urinary tract infection in South Indian type 2 diabetic subjects. Indian Journal of Nephrology. 2009; 19(3):107-111.

[17]. Ramana BV, Chaudhury A. Prevalence of uropathogens in diabetic patients and their resistance pattern at a tertiary care centre in south India. Int J Biol Med Res. 2012; 3(1):1433-1435.

[18]. 12.. Adeyeba OA, Adensiji YO, Omosigho PO. Bacterial Urinary Tract Infections in Patients with Diabetes Mellitus. International Journal of Tropical Medicine. 2007; 2(3): 89-92.30

[19]. Boyanova L, Mitov Ivan. Antibiotic Resistance Rates in Causative Agents of Infections in Diabetic Patients. Expert Rev Anti Infect Ther. 2013; 11(4): 411-420.

[20]. Casqueiro Juliana, Casqueiro Janine, Alves C. Infections in patients with diabetes mellitus: A review of pathogenesis. Indian J Endocrinol Metab. 2012; 16(1): S27-S36.

[21]. Eshwarappa M, Dosegowda R, Vrithmani AI, Khan MW, et al. Clinico-microbiological profile of urinary tract infection in South India. Indian Journal of Nephrology. 2011; 21(1): 30-36.

[22]. 33. Acharya D, Bogati B, Shrestha GT, Gyawali P. Diabetes mellitus and Urinary Tract Infection: Spectrum of Uropathogens and their Antibiotic Sensitivity Pattern. JMMIHS. 2015; 1(4): $24-28$.

[23]. Jha PK, Baral R, Khana B. Prevalence of Uropathogens in Diabetic Patients and Their Susceptibility Pattern at a Tertiary Care Center in Nepal-A Retrospective Study. International Journal of Biomedical Laboratory Science. 2014; 1.3(2): 29-34.

[24]. 34. Rawat V, Singhai M, Kumar A, Jha PK, Goyal R. Bacteriological and Resistance Profile in Isolates from Diabetic Patients. North American Journal of Medical Sciences. 2012; 4(11): 563 - 568.

[25]. Sowmya S, Lakshmidevi N. Prevalence and Incidence of Urinary Tract Infection among Diabetic Patients in Mysore. International Journal of Recent Scientific Research. 2013;

[26]. 4(10): 1651-1656

[27]. Sibi G, Devi AP, Fouzia K, Patil BR. Prevalence, Microbiologic Profile of Urinary Tract infection and its treatment with Trimethoprim in Diabetic Patients. Research Journal of Microbiology. 2011; 6(6): 543-551.

[28]. Vishal S, Vishal G, Mridula M. Prevalence Of Uropathogens In Diabetic Patients And Their Antimicrobial Susceptibility Pattern. National Journal of Laboratory Medicine. 2012; 1(1): 26-28.

[29]. Al-Qaseer A, Abdul-wahab BH, Abbas OK. Bacteriological finding of urinary tract infection in diabetic patients. International Journal of Advanced Research. 2014; 2(10): 274-279.

[30]. Njunda AL, Assob NJ C, Nsagha SD, Nde FP, et al. Uropathogens from diabetic patients with asymptomatic bacteriuria and Urinary tract infections. Scientific Journal of Microbiology. 2012; 1(6): 141-146.

[31]. Al-Rubeaan KA, Moharram O, Al-Naqeb D, Hassan A, Rafiullah MR. Prevalence of urinary tract infection and risk factors among Saudi patients with diabetes. World J Urol. 2013; 31(3):573-8.

[32]. Lyamuya EF, Moyo SJ, Komba EV, Haule M. Prevalence, Antimicrobial Resistance and Associated Risk Factors for Bacteriuria in Diabetic Women in Dar es Salaam, Tanzania. Afr. J. Microbiol. Res. 2011; 5(6): 683-689.

[33]. Gizachew Y, Daniel A, Yimtubezinash W, Chandrashekhar GU. Urinary Tract Infection: Bacterial Etiologies, Drug Resistance Profile and Associated Risk Factors in Diabetic Patients Attending Gondar University Hospital, Gondar, Ethiopia. Euro. J. Exp. Bio.

[34]. 2012; 2(4): 889-898.

[35]. A. A. Alsultan,1 B. A. Evans,2 E. A. Elsayed,1 S. I. Al-Thawadi,3A. Y. Al-Taher,1 S. G. B. Amyes,4 et al. High frequency of carbapenem-resistant Acinetobacter baumannii in patients with diabetes mellitus in Saudi Arabia.Journal of Medical Microbiology 2013: $62,885-888$ 